\title{
1 Type-2 diabetes with low LDL-C: genetic insights into a unique phenotype
}

2 Short running title: Genetics of LDL-C and type-2 diabetes

4 Yann C. Klimentidis ${ }^{1,2}{ }^{*}$, Amit Arora ${ }^{1}$, Michelle Newell ${ }^{1}$, Jin Zhou ${ }^{1}$, Jose M. Ordovas ${ }^{3,4}$,

5 Benjamin J. Renquist ${ }^{5}$, Alexis C. Wood $^{6}$

6

$7 \quad{ }^{1}$ Department of Epidemiology and Biostatistics, Mel and Enid Zuckerman College of Public

8 Health, University of Arizona, Tucson, AZ, USA

$9 \quad{ }^{2}$ BIO5 Institute, University of Arizona, Tucson, AZ, USA

$10 \quad{ }^{3}$ Nutrition and Genomics Laboratory, JM-USDA Human Nutrition Research Center on Aging

11 Tufts University, Boston, MA, USA

$12{ }^{4}$ IMDEA Food Institute, CEI UAM+CSIC, Madrid, Spain

$13{ }^{5}$ School of Animal and Comparative Biomedical Sciences, University of Arizona, Tucson, AZ, 14 USA

$15{ }^{6}$ USDA/ARS Children's Nutrition Research Center, Baylor College of Medicine. Houston, TX, 16 USA

17 * Corresponding Author: Department of Epidemiology and Biostatistics, Mel and Enid

18 Zuckerman College of Public Health, University of Arizona, Tucson, Arizona, 85724, 520-621-

19 0147, yann@email.arizona.edu

20 Word Count: 3,650 ; 2 Tables, 3 Figures 


\section{Abstract}

22 Although hyperlipidemia is traditionally considered a risk factor for type-2 diabetes (T2D),

23 evidence has emerged from statin trials and candidate gene investigations suggesting that lower

24 LDL-C increases T2D risk. We thus sought to comprehensively examine the phenotypic and

25 genotypic relationships of LDL-C with T2D. Using data from the UK Biobank, we found that

26 LDL-C was negatively associated with T2D (OR=0.43[0.41, 0.45] per mmol/L unit of LDL-C),

27 despite positive associations of LDL-C with HbAlc and BMI. We then performed the first

28 genome-wide exploration of variants simultaneously associated with lower LDL-C and increased

29 T2D risk, using data on LDL-C from the UK Biobank $(\mathrm{n}=431,167)$ and the GLGC consortium

$30(\mathrm{n}=188,577)$, and T2D from the DIAGRAM consortium $(\mathrm{n}=898,130)$. We identified 31 loci

31 associated with lower LDL-C and increased T2D, capturing several potential mechanisms. Seven

32 of these loci have previously been identified for this phenotype, and 9 have previously been

33 implicated in non-alcoholic fatty liver disease. Finally, two-sample Mendelian randomization

34 analyses suggest that low LDL-C causes T2D, although causal interpretations are challenging

35 due to pleiotropy. Our findings extend our current understanding of the higher T2D risk among

36 individuals with low LDL-C, and of the underlying mechanisms, including those underlying the

37 diabetogenic effect of LDL-C-lowering medications. 


\section{Introduction}

Rates of cardiovascular disease (CVD) and type-2 diabetes (T2D) are among the most pressing health concerns worldwide. These two diseases share many risk factors, and tend to cooccur; T2D carries a 2-4 fold increase in risk for CVD, and more than $70 \%$ of patients with T2D will die from cardiovascular complications (1). Yet, there remains controversy over whether all risk factors exert similar effects on the risk of these two conditions. Low-density lipoprotein cholesterol (LDL-C) is a class of highly atherogenic particles, and circulating levels of LDL-C are a causal risk factor for CVD across the lifespan (2). Lipid lowering medications, in particular from the statin drug class, are effective at lowering levels of LDL-C which has a dose-response relationship with a reduction in adverse cardiovascular events (3). The perception of a strong inter-relationship of T2D with hyperlipidemia, in particular higher LDL-C, has led to a standard of care in which lipid-lowering medications are a common therapeutic option for T2D. For example, the American Diabetes Association (ADA) recommends high intensity statin use for individuals with diabetes with prevalent atherosclerotic cardiovascular disease (ASCVD) or a greater than 20\% 10-year risk of ASCVD until they reach recommended stringent targets of LDL-C $<70 \mathrm{mg} / \mathrm{dL}$. The ADA further recommends moderate-intensity statin use for T2D patients 40 years and older without ASCVD (4). of T2D patients with and without CVD were on a statin, respectively (5). Similar figures have

57 emerged from analyses of Norwegian (6) and US populations (7). This almost ubiquitous use of 58 statins in T2D is highly concerning given that several lines of evidence suggest that lowered LDL-C is a causal risk factor for T2D. A small number of observational studies have reported that individuals with low levels of LDL-C (e.g. $<60 \mathrm{mg} / \mathrm{dl}$ ), exhibit a higher risk of T2D $(8,9)$, 
61 and that among individuals with coronary disease, LDL-C and T2D are inversely related (10).

62 Further, individuals with familial hypercholesterolemia exhibit a decreased risk of T2D (11).

63 Clinical trials have found that statin use conveys an increased T2D risk (odds ratio=1.09) $(12,13)$

64 in a dose-dependent manner (14). Although statins do effectively lower LDL-C, LDL is key to

65 delivering hepatic lipids from the liver to peripheral tissues. Accordingly, by lowering LDL-C

66 production, statins mute the ability of the liver to export lipids. Given that the incidence and

67 severity of hyperinsulinemia and insulin resistance is directly related to hepatic lipid concentrations, it is easy to understand how limiting hepatic lipid export may indirectly increase

69 the incidence of T2D $(15,16)$.

Genetic studies have lent further support to inverse phenotypic associations between

71 LDL-C and T2D, with recent studies pointing to genetic loci that harbor variants exerting

72 opposing effects on LDL-C and T2D. These include loci containing the HMGCR (17), APOE

73 (18,19), PCSK9 (17,19,20), NPC1L1 (17,19), PNPLA3 (19), TM6SF2 (19), GCKR (19), and

74 HNF4A (19) genes. Furthermore, both Fall et al. (21) and White et al. (22) have found that

75 genetically-predicted higher LDL-C was associated with a lower risk of T2D. Yet, genetic

76 findings show that not all variants have opposing effects on LDL-C levels and T2D risk. LDL-C

77 lowering variants in $A B C G 5 / G 8$ and $L D L R$ genes were not shown to alter T2D risk (17) and

78 subsets of LDL-C lowering alleles provide a stronger prediction against T2D than the full gamut

79 (21). LDL-C levels, like T2D, are reflective of a number of physiological processes. The findings

80 outlined above suggest that there is heterogeneity in T2D outcomes, depending on which

81 pathways are the primary LDL-C lowering mechanisms, and that genetic studies may be able to

82 give us insights into these pathways. A better understanding of which genetic loci lower LDL-C

83 but increase T2D risk may thus yield mechanistic insights that may help develop therapeutic 
84 options that lower lipid levels without raising the risk of T2D, and help identify individuals at

85 greater risk for $\mathrm{T} 2 \mathrm{D}$ with statin use.

The conclusion of the ADA's Professional Practice committee is that "the cardiovascular event rate reduction with statins far outweighed the risk of incident diabetes" due to their belief that the increase in risk "may be limited to those with diabetes risk factors". By studying genetic loci that are inversely associated (or not) with LDL-C and T2D risk, we aimed to identify pathways and therapeutic targets that may be harnessed to develop optimal therapeutic options.

Here, we first examined the relationship of directly-measured LDL-C with prevalent T2D, HbA1c, and BMI. We then sought to identify, for the first time on a genome-wide scale, loci simultaneously associated with lower LDL-C and increased T2D (and vice versa). Upon identifying variants, we sought to generate additional mechanistic insights by testing of associations with seven other traits in the UK Biobank related to T2D, LDL-C, and non-alcoholic fatty liver disease (NAFLD). Finally, we performed two-sample Mendelian randomization (MR) analyses to examine a potential causal effect of LDL-C on risk of T2D.

\section{Methods}

\section{UK Biobank}

Data from the UK Biobank was used for (1) phenotypic data analysis, which examined the associations of LDL-C and TG with T2D, HbA1c, and BMI, (2) discovery GWAS for variants which are associated with lower LDL-C and higher T2D, and (3) creation of a LDL-C genetic instrument to test in a 2-sample MR framework for a potential causal relationship between LDL-C and T2D. The UK Biobank is a prospective cohort study of approximately 
500,000 individuals between the ages of 39 and 72, living throughout the United Kingdom (UK). Participants attended one of 21 assessment centers in the UK and had their blood drawn for biomarker and genetic analysis, and weight and height measured to derive BMI $\left(\mathrm{kg} / \mathrm{m}^{2}\right)$. Directly-measured LDL-C, HbA1c, HDL, TG, alanine aminotransferase (ALT) and aspartate aminotransferase (AST) were obtained from all UK Biobank participants at the baseline visit between 2006 and 2010 in a non-fasting state. LDL-C was assessed by enzymatic protective selection analysis on a Beckman Coulter AU5800. Prevalent T2D was defined using the following criteria: 1) self-reported T2D or generic diabetes in verbal interviews, 2) over 35 years at age of diagnosis, and 3) not using insulin within one year of diagnosis, to exclude possible type-1 diabetes cases (23).

\section{UK Biobank genotypes}

Genotypes in the UK Biobank were obtained with the Affymetrix UK Biobank Axiom Array (Santa Clara, CA, USA), while $10 \%$ of participants were genotyped with the Affymetrix UK BiLEVE Axiom Array. Details regarding imputation, principal components analysis, and QC procedures are described elsewhere (24). Individuals with unusually high heterozygosity, with a high $(>5 \%)$ missing rate, or with a mismatch between self-reported and genetically-inferred sex were excluded from analyses. SNPs out of Hardy-Weinberg equilibrium $\left(\mathrm{p}<1 \times 10^{-6}\right)$, with a high missing rate $(>1.5 \%)$, with a low minor allele frequency $(<0.1 \%)$, or with a low imputation accuracy $($ info $<0.4)$ were excluded from analyses. This resulted in the availability of approximately 15 million SNPs for analysis. 
DIAGRAM and GLGC GWAS meta-analysis summary statistics

The latest GWAS meta-analysis summary statistics for T2D (unadjusted for BMI) were obtained from the DIAGRAM (Diabetes Genetics Replication and Meta-Analysis) consortium that includes data on up to 898,130 individuals (74,124 and 824,006 controls), including UK Biobank individuals (25). We used the results of our GWAS of LDL-C in UKB along with the aforementioned DIAGRAM-T2D results for the discovery of inverse association signals. We then replicated LDL-C associations of our top hits with an independent GWAS meta-analysis of LDL-C from the GLGC (26) (Global Lipids Genetics Consortium; n=188,577; this meta-analysis does not include the UK Biobank study). Across the UK Biobank, DIAGRAM, and GLGC summary statistics, we aligned all SNP alleles and their corresponding effects by using the harmonize function in the TwoSampleMR package in $\mathrm{R}(27)$.

\section{Mendelian randomization}

In a two-sample Mendelian randomization (MR) framework, we used 185 independent variants associated with LDL-C in the UK Biobank (as detailed below) as the genetic exposure, and the results of a previous DIAGRAM T2D GWAS meta-analysis (does not include the UK Biobank) as the outcome (28). The TwoSampleMR package in R was used to perform analyses. We used the clump_data function to identify independent exposure SNPs $\left(\mathrm{r}^{2}<0.001\right.$ in 1,000 Genomes Project European samples) from all SNPs with $\mathrm{p}<5 \times 10^{-8}$, and excluded unresolvable strand-ambiguous SNPs and any SNPs for which no proxy could be identified $\left(\mathrm{r}^{2}<0.8\right)$. We examined results from the inverse-variance weighted (IVW), MR-Egger, and weighted median analyses. 

and TG in the UK Biobank, we excluded all participants who self-reported (at baseline) use of

154 cholesterol-lowering medications during the touchscreen survey, or statin medication during the 155 verbal interview. To examine levels of $\mathrm{HbA1}$ c by decile of LDL-C, we excluded participants 156 who self-reported T2D at the baseline visit. We further excluded individuals with outlier values 157 of HbA1c greater than four standard deviations from the mean. Deciles were calculated using the 158 'quantcut' function in the 'gtools v3.5.0' library in R. Once deciles were established, T2D 159 prevalence by LDL-C/TG deciles was calculated and plotted with confidence intervals determined by the Clopper-Pearson interval (29). Mean HbAlc and BMI and their distributions 161 are shown in boxplots for each decile of LDL-C. We further examined T2D prevalence by LDL-

$162 \mathrm{C}$ decile separately in males and females, and in different age groups (40-49 years, 50-59 years, 163 and 60-69 years). with T2D as the outcome, and linear regression with HbA1c and BMI as outcomes. For each analysis, we used the same exclusion criteria as those mentioned above, and adjusted for 'last

167 eating' time (excluding individuals reporting extreme values, $>16$ hours), age, sex, and center.

168 To normalize residuals, we transformed LDL-C, TG cholesterol, HbA1c, and BMI by inverse 169 normalization for all linear regression analyses. In analyzing the association of LDL-C with 170 T2D, we also tested for interactions with sex and age and provided stratified analyses 171 accordingly. 

individuals on cholesterol-lowering medication was corrected by dividing it by a correction

174 factor of 0.63 (30). We transformed LDL-C by inverse normalization. We used BOLT-LMM

175 software (31) to perform GWAS on individuals of European descent $(n=431,167)$ and included

176 'last eating' time (see above), sex, age, age ${ }^{2}$, center, genotyping chip and the first 10 PCs as

177 covariates. BOLT-LMM performs a linear mixed model regression that includes a random effect

178 of all SNP genotypes other than the one being tested. We aligned effect sizes across the GWAS

179 summary statistics of each trait to the same effect allele using the harmonize function, as

180 mentioned above. We then selected only those SNPs that exhibited opposite directions of effects

181 for LDL-C and T2D. Among these, we selected only those with an association p-value $<5 \times 10^{-5}$

182 for LDL-C and for T2D in a region defined as a maximum size of $500 \mathrm{~kb}$. At this p-value

183 threshold, the prior probability of a given SNP associated with two traits and with discordant

184 direction of effect under the null hypothesis, corresponds to $0.00005 \times 0.000025=1.25 \times 10^{-9}$

185 (32). We did not employ a multivariate approach because current methods do not typically report trait-specific effect size estimates (or directions). Although MTAG (33) does provide effect size

187 estimates for each trait, this method is not suitable for traits with low genetic correlation, which

188 is the case for LDL-C and T2D (25). For the replication of the discovered loci, we used a

189 threshold of $\mathrm{p}<5 \times 10^{-3}$ in GLGC (does not include UK Biobank individuals), and exhibiting the 190 opposite direction of association with T2D in DIAGRAM. To test the association of the 31 SNPs 191 (T2D increasing allele) that we identified with a range of other cardiometabolic traits, we used 192 similar methods described above for the LDL-C GWAS. For TG and HDL-C, we excluded 193 individuals reporting cholesterol-lowering medication. For ALT and AST, we excluded 15,138 194 individuals with medical conditions, other than NAFLD, that could affect liver enzyme levels 
195 (34). For HbA1c, we excluded individuals with self-reported T2D (see above). For WHR, we

196 additionally adjusted for BMI prior to inverse normalization and subsequent GWAS. We inverse

197 normalized all traits before GWAS. After association of 31 SNPs with each of these seven

198 additional phenotypes, we normalized the effect sizes by dividing the beta coefficients by the

199 corresponding standard errors, and dividing by the square root of the respective sample size. We

200 then used hierarchical clustering to group the identified variants according to their pattern of

201 association with all nine traits, including T2D and LDL-C. We used the hclust function in R,

202 with the Euclidian metric to calculate distances, and the Ward clustering method (35).

\section{Results}

Participant characteristics

In a sample size of 379,617 individuals after exclusions of individuals on lipid-lowering

207 medication, T2D prevalence was $0.9 \%$, and was higher in males $(1.53 \%)$ than females $(0.63 \%)$.

208 Individuals with T2D had lower LDL-C, higher TG, higher HbA1c, and higher BMI (see

209 Supplementary Table 1).

213 prevalence $\left.=0.90 \% ; \mathrm{OR}=0.43[0.41,0.45], \mathrm{p}=2.6 \times 10^{-269}\right)$. Individuals in the lowest decile of

214 LDL-C exhibited the highest prevalence of T2D and a consistent decrease in T2D prevalence

215 was observed with increasing LDL-C (see Figure 1). We found a very similar negative 
association of LDL-C with T2D among only the individuals reporting the use of cholesterollowering medication. We found a significant interaction of LDL-C with sex $\left(p=1.2 \times 10^{-18}\right)$, whereby the association of LDL-C with T2D was stronger among men $(\mathrm{OR}=0.36[0.34,0.38]$, $\left.\mathrm{p}=3.6 \times 10^{-227}\right)$ than among women $\left(\mathrm{OR}=0.54[0.50,0.58], \mathrm{p}=3.63 \times 10^{-60}\right)($ see Supplementary Table 2 and Supplementary Figure 1). We also observed a stronger inverse association between LDL-C and T2D prevalence among older individuals (Supplementary Table 2 and Supplementary Figure 2). Positive associations were found between LDL-C and both HbA1c (after exclusion of individuals with T2D; $\beta=0.14$, se $\left.=0.0017, \mathrm{p}<2.2 \times 10^{-308}\right)$ and $\mathrm{BMI}(\beta=$ $\left.0.16, \mathrm{se}=0.0016, \mathrm{p}<2.2 \times 10^{-308}\right)($ Figure 1 and Supplementary Table 2$)$. We also observed a positive association between $\mathrm{TG}$ and $\mathrm{T} 2 \mathrm{D}$ prevalence $\left(\mathrm{OR}=1.35[1.32,1.39], \mathrm{p}=2.4 \times 10^{-134}\right.$; Supplementary Table 2 and Supplementary Figure 3).

\section{Loci associated inversely with LDL-C and T2D}

We identified 48 loci associated in opposite directions with LDL-C and T2D using the UK Biobank LDL-C and the DIAGRAM T2D results. Among these, 31 replicated with respect to LDL-C association when using the GLGC LDL-C GWAS results instead of UK Biobank (see Table 1). Several loci are previously known or suspected to be inversely associated with LDL-C and T2D (HMGCR, APOE, NPC1L1, PNPLA3, TM6SF2, GCKR, and HNF4A). However, most of the loci are novel for this LDL-T2D trait. Of these novel loci, 12 have previously been identified for LDL-C in the GLGC GWAS, and 14 were previously identified in T2D GWAS, and 14 have not been identified previously with either trait. The loci with the strongest degree of opposing effects include FNDC7-STXBP3, SORT1-PSMA5, HMGCR-POC5, PPP1R3B, and GCKR (see Figure 2). 
The variants that we have identified can be linked with genes that affect de novo fatty

240

241

242

243

244

245

246

247

248

249

250

251

252

253

254

255

256

257

258

259

260 acid synthesis, hepatic lipid uptake, hepatic lipid export, peripheral tissue lipid balance, fatty liver of unknown origin, insulin secretion, and insulin action (see Supplementary Table 4). They are associated in distinct patterns across a range of cardiometabolic traits (see Figure 3 ). At these loci, the T2D-increasing alleles at the SNPs with lowest T2D p-value are generally associated with higher HbA1c levels, lower HDL levels, and a higher WHR, although this pattern is not entirely consistent across all 31 SNPs. A cluster of $A P O E, G C K R$, and TM6SF2 emerged showing a pattern of lower TG, higher HbAlc, and generally higher BMI and WHR. Another cluster includes ERLIN1-CWF19L1 and PNPLA3 that exhibits lower HDL, and higher liver enzymes. Finally, another cluster emerged including SORT1-PSMA5, HMGCR-POC5, and HNF4A, which generally exhibit higher BMI and lower HDL (see Figure 3).

\section{Mendelian randomization}

We used 140 independent SNPs significantly associated with LDL-C in the UK Biobank as the genetic instrument. We found a consistent negative association with T2D across all MR methods implemented (IVW OR $=0.82[0.72,0.93]$ ) except for simple mode, although we noted a high degree of heterogeneity (see Table 2 and Supplementary Figures 4-6).

\section{Discussion}

We used the largest sample to date to demonstrate that LDL-C is inversely correlated with T2D risk, such that individuals with low LDL-C exhibit a higher prevalence of T2D. Then, in the first genome-wide analysis aimed at identifying variants associated with both lower LDL- 
261 C and higher T2D risk, we identified 24 novel loci exerting opposite-direction effects on these

262 traits. Our analyses lend weight to the notion that the association between lower LDL-C and

263 increased T2D risk is driven, at least in part, by a specific group of genetic variants that may be

264 implicated via diverse mechanisms including hepatic lipid synthesis, export, and uptake, as well

265 as insulin secretion and action. These variants provide insight into the heterogeneous outcomes

266 for different lipid and glucose metabolism pathways and may also point to novel susceptibility

267 loci for hyperlipidemia and T2D.

We find that low LDL-C is associated with greater T2D risk, which is consistent with two

269 previous studies examining T2D prevalence (8) and incidence (9). In addition, we find that lower

270 LDL-C is associated with lower HbA1c (among individuals without T2D) and lower BMI. On

271 the other hand, we do observe a similar inverse relationship of LDL-C with T2D in the set of

272 people on cholesterol-lowering medication. We also find that unlike LDL-C, TG levels are

273 positively associated with T2D prevalence. This opposing relationship of LDL-C and TG with

274 T2D suggests that LDL are being overfilled in individuals with T2D.

Previous research into loci that jointly alter the risk for LDL-C and T2D has focused on

276 the genomic targets of lipid-lowering medications, in the hope that these analyses will give

277 specific insights into associated T2D risk. Our analyses confirmed that variants in $H M G C R$ (36)

278 and NPC1L1 (19) are associated with lower LDL-C and increased T2D risk. On the other hand,

279 our analyses did not identify variants at PCSK9. The lowest T2D p-value was 0.003 in this

280 region for a SNP with opposite direction coefficient. However, our analyses identified a fourth

281 target of lipid-lowering medications: variants in the Peroxisome Proliferator-Activated Receptor

$282(P P A R G)$ gene, the target of fibrates and thiazolidinediones. 
We observed nine variants previously identified as being associated with NAFLD:

PNPLA3, GCKR, TM6SF2, PPP1R3B, ERLIN1-CWF19L1, REEP3, HNF1A, SLC2A2, and

MICAL3 (37-39). This enrichment for NAFLD-related genes may reflect increased synthesis and storage of TG and reduced export/secretion of VLDL, leading to reduced circulating LDL-C. Indeed, the LDL-C-decreasing alleles at most of these loci are associated with increased liver enzymes, indicative of hepatic steatosis (with the exception of GCKR and $S L C 2 A 2$, consistent with a previous finding (40)). The degree of NAFLD is directly linked to the incidence and severity of hyperinsulinemia and insulin resistance in obesity $(15,16)$. In turn, lower LDL-C with increased liver enzymes would be expected to indicate increased NAFLD and T2D. A recent bidirectional Mendelian randomization study provides support for this hypothesized causal effect of NAFLD on T2D (41). Our findings that liver fat may be an important mediator of the effect of

294 cholesterol-lowering on T2D is consistent with a report showing that liver fat may help identify 295 statin-taking individuals at risk of T2D (42). Finally, it is noteworthy that the HMGCR variant 296 that lowers LDL-C is not associated with any significant change in liver enzymes, potentially 297 reflecting the lack of an increase in NAFLD incidence seen with statin medications (43).

299 metabolism. Sortilin 1 (SORT1) is highly expressed in adipocytes, and the sortilin gene product 300 facilitates the formation and export of VLDL from the liver $(44,45)$. The role of SORT1 in T2D 301 risk is not well understood. Sortilin 1 is required for insulin-dependent glucose uptake (46-48), 302 yet Sortl knockout mice may show reduced glucose and glycolic intermediates in the fasted state 303 (49). This highlights again the potential for heterogeneous paths in T2D risk and the dependence 304 on multiple pathways of lipid and glucose metabolism to explain our findings. 
Several loci were also identified which are known to be related to T2D, without known associations with LDL-C. These include THADA, C2CD4A, CENPW, and SLC12A8 (25). In addition, we identified several variants associated with lower LDL-C but increased T2D risk with no known biological pathways linking these loci to either trait. $S L C 2 A 2$, which encodes the glucose transporter 2, has not previously been associated with either LDL-C or T2D in the large respective GWAS consortia. However, GLUT2 is key to hepatic glucose uptake following a meal and the associated hepatic de novo lipogenesis (50). In fact, liver-specific GLUT2 knockout

312 decreases liver triglyceride concentrations. Importantly, GLUT2 expression in the $\beta$-cell is 313 required for the glucose stimulated insulin response (51). In turn, a locus that decreases GLUT2 expression would be expected to limit serum insulin, increase HbA1c, and decrease LDL-C. which limits inferences related to the direction of causality. As incident T2D cases develop in the UK Biobank, it will be important to examine the association of LDL-C at baseline with incident T2D. It is also difficult to identify the causal gene at identified loci. Although we annotated these

319 loci according to nearby genes and/or previous annotation, the listed genes may not necessarily 320 be directly implicated, if at all. Finally, it is difficult to draw any firm conclusions from MR as 321 these traits are highly intertwined and the assumption of no pleiotropy is unrealistic.

323 further study is warranted. We have identified a collection of genetic variants that may provide

324 insight into the mechanisms underlying the diabetogenic risk of low LDL-C, and of lipid-

325 lowering medications, and the decreased T2D risk among individuals with familial 
329 The authors would like to acknowledge the vital contributions of the GLGC and DIAGRAM

330 consortia, as well as all organizers and participants of individual participating studies. This

331 research was conducted using the UK Biobank Resource under Application Number 15678. We

332 thank the participants and organizers of the UK Biobank. The authors would like to acknowledge 333 support from the National Heart, Lung, and Blood Institutes (R01-HL136528). Dr. Wood's role

334 on this project was funded, in part, by USDA/ARS cooperative agreement \# 58-3092-5-001. The

335 contents of this publication do not necessarily reflect the views or policies of the U.S.

336 Department of Agriculture, nor does mention of trade names, commercial products, or

337 organizations imply endorsement by the U.S. Government. The funders had no role in study

338 design, data collection and analysis, decision to publish, or preparation of the manuscript.

340 The authors have no relevant conflicts of interest to disclose.

342 Author Contributions: YK conceived and designed the study. AA, MN, and YK performed

343 data analyses. YK, AW, MN, and BR wrote the manuscript. AW, BR, and JO contributed to

344 writing of the introduction and discussion; all authors read and edited the manuscript. 


\section{References}

346 1. Laakso M. Cardiovascular disease in type 2 diabetes: challenge for treatment and

347 prevention. J Intern Med. 2001 Mar;249(3):225-35.

2. Postmus I, Deelen J, Sedaghat S, Trompet S, de Craen AJM, Heijmans BT, et al. LDL cholesterol still a problem in old age? A Mendelian randomization study. Int J Epidemiol. 2015 Apr;44(2):604-12.

3. Boekholdt SM, Arsenault BJ, Mora S, Pedersen TR, LaRosa JC, Nestel PJ, et al. Association of LDL cholesterol, non-HDL cholesterol, and apolipoprotein B levels with risk of cardiovascular events among patients treated with statins: a meta-analysis. JAMA. 2012 Mar;307(12):1302-9.

4. 10. Cardiovascular Disease and Risk Management: Standards of Medical Care in Diabetes-2019. Diabetes Care. 2019 Jan;42(Suppl 1):S103-23.

5. McGurnaghan S, Blackbourn LAK, Mocevic E, Haagen Panton U, McCrimmon RJ, Sattar a contemporary analysis. Diabet Med. 2019 Jun;36(6):718-25.

6. Gjelsvik B, Tran AT, Berg TJ, Bakke A, Mdala I, Nokleby K, et al. Exploring the relationship between coronary heart disease and type 2 diabetes: a cross-sectional study of secondary prevention among diabetes patients. BJGP open. 2019 Apr;3(1):bjgpopen18X101636.

364 7. Hammad MA, Syed Sulaiman SA, Aziz NA, Mohamed Noor DA. Prescribing statins 
J Res Med Sci. 2019;24:15.

367 8. Feng QP, Wei WQ, Chung CP, Levinson RT, Sundermann AC, Mosley JD, et al. study using electronic health records. PLoS Med. 2018;15(8):1-14.

371 9. Andersson C, Lyass A, Larson MG, Robins SJ, Vasan RS. Low-density-lipoprotein cholesterol concentrations and risk of incident diabetes: epidemiological and genetic insights from the Framingham Heart Study. Diabetologia. 2015;58(12):2774-80.

10. Sacks FM, Tonkin AM, Craven T, Pfeffer MA, Shepherd J, Keech A, et al. Coronary heart disease in patients with low LDL-cholesterol: benefit of pravastatin in diabetics and enhanced role for HDL-cholesterol and triglycerides as risk factors. Circulation. 2002

11. Besseling J, Kastelein JJP, Defesche JC, Hutten BA, Hovingh GK. Association between familial hypercholesterolemia and prevalence of type 2 diabetes mellitus. JAMA. 2015 Mar;313(10):1029-36. of incident diabetes: a collaborative meta-analysis of randomised statin trials. Lancet. 2010;375(9716):735-42. and risk of developing diabetes: results from the Diabetes Prevention Program. BMJ open diabetes Res care. 2017;5(1):e000438. 
387 14. Preiss D, Seshasai SRK, Welsh P, Murphy SA, Ho JE, Waters DD, et al. Risk of incident diabetes with intensive-dose compared with moderate-dose statin therapy: a meta-analysis. JAMA. 2011;305(24):2556-64.

15. Wainwright $\mathrm{P}$, Byrne CD. Bidirectional Relationships and Disconnects between NAFLD and Features of the Metabolic Syndrome. Int J Mol Sci. 2016 Mar;17(3):367.

16. Fabbrini E, Sullivan S, Klein S. Obesity and nonalcoholic fatty liver disease: biochemical, metabolic, and clinical implications. Hepatology. 2010 Feb;51(2):679-89. between low-density lipoprotein cholesterol-lowering genetic variants and risk of type 2 diabetes: A meta-analysis. JAMA - J Am Med Assoc. 2016;

18. Zhao W, Rasheed A, Tikkanen E, Lee J-J, Butterworth AS, Howson JMM, et al. Identification of new susceptibility loci for type 2 diabetes and shared etiological pathways with coronary heart disease. Nat Genet. 2017 Oct;49(10):1450-7. association study of plasma lipids in >300,000 individuals. Nat Genet. 2017 Dec;49(12):1758-66.

20. Schmidt AF, Swerdlow DI, Holmes M V, Patel RS, Fairhurst-Hunter Z, Lyall DM, et al. PCSK9 genetic variants and risk of type 2 diabetes: a mendelian randomisation study. lancet Diabetes Endocrinol. 2017 Feb;5(2):97-105. 
22. White J, Swerdlow DI, Preiss D, Fairhurst-Hunter Z, Keating BJ, Asselbergs FW, et al. Association of lipid fractions with risks for coronary artery disease and diabetes. JAMA Cardiol. 2016;1(6):692-9.

23. Yaghootkar H, Lotta LA, Tyrrell J, Smit RAJ, Jones SE, Donnelly L, et al. Genetic Evidence for a Link Between Favorable Adiposity and Lower Risk of Type 2 Diabetes, Hypertension, and Heart Disease. Diabetes. 2016 Aug 1;65(8):2448 LP - 2460.

24. Bycroft C, Freeman C, Petkova D, Band G, Elliott LT, Sharp K, et al. The UK Biobank resource with deep phenotyping and genomic data. Nature. 2018;562(7726):203-9.

25. Mahajan A, Taliun D, Thurner M, Robertson NR, Torres JM, Rayner NW, et al. Finemapping type 2 diabetes loci to single-variant resolution using high-density imputation and islet-specific epigenome maps. Nat Genet. 2018;50(11):1505-13.

26. Willer CJ, Schmidt EM, Sengupta S, Peloso GM, Gustafsson S, Kanoni S, et al. Discovery and refinement of loci associated with lipid levels. Vol. 45, Nature genetics. 2013. p. 1274-83.

27. Hemani G, Zheng J, Elsworth B, Wade KH, Haberland V, Baird D, et al. The MR-Base platform supports systematic causal inference across the human phenome. Loos R, editor. Elife. 2018;7:e34408.

28. Consortium DiaGRAM (DIAGRAM), Consortium AGENT 2 D (AGEN-T, Consortium SAT 2 D (SAT2D), Consortium MAT 2 D (MAT2D), Consortium T 2 DGE by N sequencing in multi-ES (T2D-G, Mahajan A, et al. Genome-wide trans-ancestry metaanalysis provides insight into the genetic architecture of type 2 diabetes susceptibility. Nat Genet. 2014 Mar;46(3):234-44. 
29. CLOPPER CJ, PEARSON ES. THE USE OF CONFIDENCE OR FIDUCIAL LIMITS ILLUSTRATED IN THE CASE OF THE BINOMIAL. Biometrika. 1934 Dec $1 ; 26(4): 404-13$.

30. Nissen SE, Tuzcu EM, Schoenhagen P, Crowe T, Sasiela WJ, Tsai J, et al. Statin therapy, LDL cholesterol, C-reactive protein, and coronary artery disease. N Engl J Med. 2005 Jan;352(1):29-38.

31. Loh P-R, Kichaev G, Gazal S, Schoech AP, Price AL. Mixed-model association for biobank-scale datasets. Vol. 50, Nature genetics. United States; 2018. p. 906-8.

32. Lotta LA, Gulati P, Day FR, Payne F, Ongen H, van de Bunt M, et al. Integrative genomic analysis implicates limited peripheral adipose storage capacity in the pathogenesis of human insulin resistance. Nat Genet. 2017 Jan;49(1):17-26.

33. Turley P, Walters RK, Maghzian O, Okbay A, Lee JJ, Fontana MA, et al. Multi-trait analysis of genome-wide association summary statistics using MTAG. Nat Genet. 2018 Feb;50(2):229-37.

34. Pratt DS, Kaplan MM. Evaluation of abnormal liver-enzyme results in asymptomatic patients. N Engl J Med. 2000 Apr;342(17):1266-71.

35. Murtagh F. Multidimensional clustering algorithms. COMPSTAT Lectures 4. Lectures in Computational Statistics. 1985.

36. Higuchi S, Izquierdo MC, Haeusler RA. Unexplained reciprocal regulation of diabetes and lipoproteins. Curr Opin Lipidol. 2018 Jun;29(3):186-93.

37. Speliotes EK, Yerges-Armstrong LM, Wu J, Hernaez R, Kim LJ, Palmer CD, et al. 
Genome-wide association analysis identifies variants associated with nonalcoholic fatty liver disease that have distinct effects on metabolic traits. PLoS Genet. 2011 Mar;7(3):e1001324.

38. Yuan X, Waterworth D, Perry JRB, Lim N, Song K, Chambers JC, et al. PopulationBased Genome-wide Association Studies Reveal Six Loci Influencing Plasma Levels of Liver Enzymes. Am J Hum Genet. 2008;83(4):520-8.

39. Chambers JC, Zhang W, Sehmi J, Li X, Wass MN, Van der Harst P, et al. Genome-wide association study identifies loci influencing concentrations of liver enzymes in plasma. Nat Genet. 2011 Oct;43(11):1131-8.

40. Sliz E, Sebert S, Würtz P, Kangas AJ, Soininen P, Lehtimäki T, et al. NAFLD risk alleles in PNPLA3, TM6SF2, GCKR and LYPLAL1 show divergent metabolic effects. Hum Mol Genet. 2018;27(12):2214-23.

41. De Silva NMG, Borges MC, Hingorani AD, Engmann J, Shah T, Zhang X, et al. Liver Function and Risk of Type 2 Diabetes: Bidirectional Mendelian Randomization Study. Diabetes. 2019 Aug;68(8):1681-91.

42. Shah R V., Allison MA, Lima JAC, Bluemke DA, Abbasi SA, Ouyang P, et al. Liver fat, statin use, and incident diabetes: The Multi-Ethnic Study of Atherosclerosis. Atherosclerosis. 2015 Sep 1;242(1):211-7.

43. Sigler MA, Congdon L, Edwards KL. An Evidence-Based Review of Statin Use in Patients With Nonalcoholic Fatty Liver Disease. Clin Med Insights Gastroenterol. 2018;11:1179552218787502. 
472 44. Chen C, Li J, Matye DJ, Wang Y, Li T. Hepatocyte Sortilin 1 knockout and treatment

473

474

475

476

477

478

479

480

481

482

483

484

485

486

487

488

489

490

491

492

with a Sortilin 1 inhibitor reduced plasma cholesterol in Western diet-fed mice. J Lipid

Res. 2019 Jan 22;60(3):539-49.

45. Brown MS, Goldstein JL. Sterol regulatory element binding proteins (SREBPs):

controllers of lipid synthesis and cellular uptake. Nutr Rev. 1998 Feb;56(2 Pt 2):S1-3;

discussion S54-75.

46. Shi J, Kandror K V. Sortilin is essential and sufficient for the formation of Glut4 storage vesicles in 3T3-L1 adipocytes. Dev Cell. 2005 Jul;9(1):99-108.

47. Huang G, Buckler-Pena D, Nauta T, Singh M, Asmar A, Shi J, et al. Insulin responsiveness of glucose transporter 4 in 3T3-L1 cells depends on the presence of sortilin. Mol Biol Cell. 2013 Oct;24(19):3115-22.

48. Tsuchiya Y, Hatakeyama H, Emoto N, Wagatsuma F, Matsushita S, Kanzaki M. Palmitate-induced down-regulation of sortilin and impaired GLUT4 trafficking in C2C12 myotubes. J Biol Chem. 2010 Nov;285(45):34371-81.

49. Li J, Matye DJ, Wang Y, Li T. Sortilin 1 knockout alters basal adipose glucose metabolism but not diet-induced obesity in mice. FEBS Lett. 2017 Apr;591(7):1018-28.

50. Seyer P, Vallois D, Poitry-Yamate C, Schutz F, Metref S, Tarussio D, et al. Hepatic glucose sensing is required to preserve beta cell glucose competence. J Clin Invest. 2013 Apr;123(4):1662-76.

51. Guillam MT, Dupraz P, Thorens B. Glucose uptake, utilization, and signaling in GLUT2null islets. Diabetes. 2000 Sep;49(9):1485-91. 
bioRxiv preprint doi: https://doi.org/10.1101/837013; this version posted November 13, 2019. The copyright holder for this preprint (which was not certified by peer review) is the author/funder, who has granted bioRxiv a license to display the preprint in perpetuity. It is made available under aCC-BY-NC-ND 4.0 International license. 
495 Table 1: List of genetic loci in which all SNPs have $\mathrm{p}<5 \mathrm{E}-5$ for both LDL-C and T2D and with 496 opposite directions of effect, for UK Biobank LDL-C and DIAGRAM T2D. Beta coefficients 497 refer to the SNP with the lowest $\mathrm{p}$-value for each trait in the respective region. The minimum $\mathrm{p}$ 498 value for LDL-C in GLGC summary statistics for the region discovered in UK Biobank is also 499 shown.

\begin{tabular}{|c|c|c|c|c|c|c|c|c|c|c|}
\hline \multicolumn{10}{|c|}{ UK Biobank LDL-C and DIAGRAM T2D } & \multirow{2}{*}{$\frac{\text { GLGC LDL-C }}{\text { p_minP_LDL }}$} \\
\hline Chr & bp_min & bp_max & SNP_minP_LDL & SNP_minP_T2D & Nearest Gene(s) & beta_minP_LDL & beta_minP_T2D & P_minP_LDL & P_minP_T2D & \\
\hline 1 & $109,285,825$ & $109,285,825$ & rs12144658 & rs12144658 & FNDC7-STXBP3 & 0.028 & -0.095 & 7.00E-06 & $8.30 \mathrm{E}-06$ & $1.67 \mathrm{E}-03$ \\
\hline 1 & $109,843,746$ & $110,120,221$ & rs56218586 & rs41279732 & SORT1-PSMA5 & -0.095 & -0.061 & $2.60 \mathrm{E}-118$ & $1.40 \mathrm{E}-06$ & $6.31 \mathrm{E}-27$ \\
\hline 2 & $27,161,476$ & $28,246,841$ & rs1260326 & rs1260326 & GCKR & 0.053 & -0.067 & $2.60 \mathrm{E}-165$ & $1.30 \mathrm{E}-24$ & $8.35 \mathrm{E}-10$ \\
\hline 2 & $43,452,183$ & $44,020,441$ & rs11901691 & rs116097055 & THADA & 0.012 & -0.180 & 4.10E-10 & $1.50 \mathrm{E}-10$ & $1.11 \mathrm{E}-03$ \\
\hline 2 & $121,306,440$ & $121,350,667$ & rs17050272 & rs10460461 & LINC01101-GLI2 & -0.026 & -0.043 & $6.40 \mathrm{E}-42$ & $1.40 \mathrm{E}-11$ & 8.93E-09 \\
\hline 2 & $234,508,963$ & $234,565,917$ & rs2741029 & rs2741042 & UGT1A8 & 0.009 & 0.030 & $3.50 \mathrm{E}-05$ & $1.40 \mathrm{E}-05$ & 3.54E-06 \\
\hline 3 & $12,058,246$ & $12,456,689$ & rs17036170 & rs17036170 & PPARG & -0.073 & 0.130 & $9.90 \mathrm{E}-15$ & $2.70 \mathrm{E}-06$ & $1.49 \mathrm{E}-08$ \\
\hline 3 & $124,912,207$ & $125,122,081$ & rs9873519 & rs649961 & SLC12A8 & -0.011 & 0.038 & $1.90 \mathrm{E}-08$ & $1.30 \mathrm{E}-09$ & 7.83E-04 \\
\hline 3 & $170,631,635$ & $170,743,747$ & rs7620985 & rs5402 & $S L C 2 A 2$ & 0.024 & -0.051 & $2.90 \mathrm{E}-13$ & $1.30 \mathrm{E}-07$ & 4.19E-05 \\
\hline 5 & $74,352,180$ & $75,042,913$ & rs2112653 & rs2307111 & HMGCR-POC5 & -0.069 & 0.053 & 1.00E-200 & 3.30E-16 & $2.97 \mathrm{E}-86$ \\
\hline 5 & $78,350,390$ & $78,385,845$ & rs10065798 & rs10944 & ВНMT2 & -0.008 & 0.032 & $1.10 \mathrm{E}-05$ & $6.80 \mathrm{E}-07$ & $2.98 \mathrm{E}-03$ \\
\hline 6 & $31,145,920$ & $31,372,569$ & rs2523535 & rs887464 & $H L A-C / B$ & 0.013 & 0.032 & $1.20 \mathrm{E}-11$ & $9.30 \mathrm{E}-07$ & $2.49 \mathrm{E}-11$ \\
\hline 6 & $32,127,477$ & $33,514,567$ & rs9273410 & rs17613292 & $H L A-D Q A 1 / B 1$ & -0.020 & 0.063 & $8.10 \mathrm{E}-23$ & $1.80 \mathrm{E}-16$ & $3.63 \mathrm{E}-15$ \\
\hline 6 & $126,629,425$ & $127,167,072$ & rs9388490 & rs11759026 & CENPW & -0.015 & -0.067 & $3.20 \mathrm{E}-15$ & $1.30 \mathrm{E}-18$ & $3.83 \mathrm{E}-03$ \\
\hline 7 & $44,585,052$ & $44,709,372$ & rs2008428 & rs79064806 & NPC1L1-DDX56 & -0.038 & -0.049 & $4.50 \mathrm{E}-63$ & $7.10 \mathrm{E}-06$ & $6.32 \mathrm{E}-22$ \\
\hline 7 & $100,266,081$ & $100,414,386$ & rs221793 & rs564449 & GIGYF1-POP7-EPO & 0.029 & -0.048 & $6.70 \mathrm{E}-20$ & $2.20 \mathrm{E}-06$ & 4.77E-04 \\
\hline 8 & $9,168,146$ & $9,740,317$ & rs9987289 & rs17662402 & $P P P 1 R 3 B$ & -0.057 & 0.086 & $1.10 \mathrm{E}-65$ & $9.10 \mathrm{E}-10$ & $1.41 \mathrm{E}-27$ \\
\hline 10 & $64,988,931$ & $65,340,897$ & rs3847326 & rs12245367 & REEP3 & 0.012 & 0.028 & $9.20 \mathrm{E}-11$ & $1.30 \mathrm{E}-05$ & $1.12 \mathrm{E}-03$ \\
\hline 10 & $101,900,579$ & $102,049,300$ & rs12784396 & rs10883451 & ERLIN1-CWF19L1 & 0.012 & 0.032 & 1.10E-10 & 3.50E-07 & $1.82 \mathrm{E}-03$ \\
\hline 11 & $65,422,853$ & $65,604,195$ & rs4930163 & rs56019505 & OVOL1-SNX32 & 0.012 & 0.041 & $2.50 \mathrm{E}-06$ & $1.20 \mathrm{E}-05$ & 4.29E-04 \\
\hline 12 & $50,881,148$ & $50,987,378$ & rs7316864 & rs34867510 & DIP2B & -0.011 & -0.029 & $6.70 \mathrm{E}-08$ & $3.20 \mathrm{E}-05$ & 1.09E-06 \\
\hline 12 & $121,435,778$ & $121,463,140$ & rs11065388 & rs 12231806 & HNF1A-OASL & 0.018 & 0.050 & $1.30 \mathrm{E}-10$ & 8.00E-08 & 4.36E-06 \\
\hline 15 & $62,383,155$ & $62,399,541$ & rs4502156 & rs8037894 & $C 2 C D 4 A / B$ & -0.008 & -0.047 & 1.30E-05 & $3.70 \mathrm{E}-13$ & 1.05E-04 \\
\hline 17 & $29,628,549$ & $29,749,444$ & rs7502556 & rs1129506 & NF1-EVI2A & -0.014 & -0.035 & $2.80 \mathrm{E}-13$ & $8.80 \mathrm{E}-08$ & $5.81 \mathrm{E}-06$ \\
\hline 17 & $46,044,031$ & $46,114,620$ & rs12935890 & rs1076236 & COPZ2 & -0.015 & -0.031 & $6.20 \mathrm{E}-13$ & $2.80 \mathrm{E}-06$ & $3.64 \mathrm{E}-03$ \\
\hline 19 & $19,320,825$ & $19,793,545$ & rs2228603 & rs8107974 & TM6SF2 & -0.108 & -0.093 & $1.00 \mathrm{E}-200$ & $6.30 \mathrm{E}-15$ & 4.60E-61 \\
\hline 19 & $45,324,756$ & $45,428,234$ & rs147711004 & rs429358 & $A P O E$ & 0.170 & 0.080 & $1.00 \mathrm{E}-200$ & $1.80 \mathrm{E}-18$ & $0.00 \mathrm{E}+00$ \\
\hline 19 & $46,123,929$ & $46,314,504$ & rs2238689 & rs9797621 & GIPR & 0.017 & -0.057 & $9.40 \mathrm{E}-19$ & $1.70 \mathrm{E}-17$ & $1.86 \mathrm{E}-09$ \\
\hline 20 & $42,958,768$ & $43,042,364$ & rs1800961 & rs1800961 & HNF4A & -0.076 & 0.160 & $1.80 \mathrm{E}-42$ & $3.20 \mathrm{E}-20$ & $5.16 \mathrm{E}-11$ \\
\hline 22 & $18,415,158$ & $18,486,514$ & rs11703382 & rs5992928 & MICAL3 & 0.011 & 0.035 & $5.50 \mathrm{E}-07$ & $3.10 \mathrm{E}-06$ & $3.42 \mathrm{E}-05$ \\
\hline 22 & $44,324,855$ & $44,394,402$ & rs2294915 & rs3747207 & PNPLA3 & -0.014 & 0.049 & 5.70E-10 & 2.10E-10 & 2.91E-05 \\
\hline
\end{tabular}

500 bp_min \& bp_max: minimum and maximum base pair position for which all SNPs have $\mathrm{p}<5 \mathrm{E}-5$ for LDL-C and 501 T2D, and with opposite directions of effect. Human build GRCh37/hg19; Beta coefficients correspond to log-odds 502 ratios for T2D, and standard deviations for LDL-C. P-values from GLGC LDL-C associations are for SNPs showing 503 opposite direction of association with T2D (DIAGRAM). 
504 Table 2: Results of Mendelian randomization of LDL-C on type-2 diabetes.

\begin{tabular}{cccccc}
\hline Method & n SNP & OR [95\% CI] & p-value & $\begin{array}{c}\text { Modified } \\
\text { Cochran's Q, } \\
\text { p-value }\end{array}$ & $\begin{array}{c}\text { MR-Egger } \\
\text { Y-intercept, } \\
\text { p-value }\end{array}$ \\
\hline MR Egger & 140 & $0.68[0.54,0.85]$ & $8.58 \mathrm{E}-04$ & $1.01 \mathrm{E}-04$ & 0.05 \\
Weighted median & 140 & $0.79[0.66,0.96]$ & $1.98 \mathrm{E}-02$ & & \\
Inverse variance weighted & 140 & $0.82[0.72,0.93]$ & $2.64 \mathrm{E}-03$ & $4.02 \mathrm{E}-05$ & \\
Simple mode & 140 & $0.79[0.52,1.21]$ & $2.80 \mathrm{E}-01$ & & \\
Weighted mode & 140 & $0.81[0.68,0.96]$ & $1.82 \mathrm{E}-02$ & & \\
\hline
\end{tabular}

505 


\section{Figure Legends:}

507 Figure 1: T2D prevalence, HbA1c, and BMI by LDL-C decile in the UK Biobank. T2D 508 prevalence is shown as a percentage with error bars corresponding to the Clopper-Pearson 509 confidence interval. Whisker plots show the median value (horizontal line in box), the $25^{\text {th }}$ and $51075^{\text {th }}$ percentile delimited by the box, and the vertical lines extending to the $5^{\text {th }}$ and $95^{\text {th }}$ percentile.

512 Figure 2: Plot of beta coefficients for LDL-C vs. T2D for SNPs with opposite directions of 513 effect on these two traits. Beta coefficients correspond to log-odds ratios for T2D, and standard 514 deviations for LDL-C.

516 Figure 3: Association of T2D increasing allele at 31 identified SNPs with nine cardiometabolic 517 traits, and clustering dendogram based on corresponding standardized effect sizes. 
519 Figure 1: T2D prevalence, HbA1c, and BMI by LDL-C decile in the UK Biobank. T2D

520 prevalence is shown as a percentage with error bars corresponding to the Clopper-Pearson

521 confidence interval. Whisker plots show the median value (horizontal line in box), the $25^{\text {th }}$ and

$52275^{\text {th }}$ percentile delimited by the box, and the vertical lines extending to the $5^{\text {th }}$ and $95^{\text {th }}$ percentile.

523
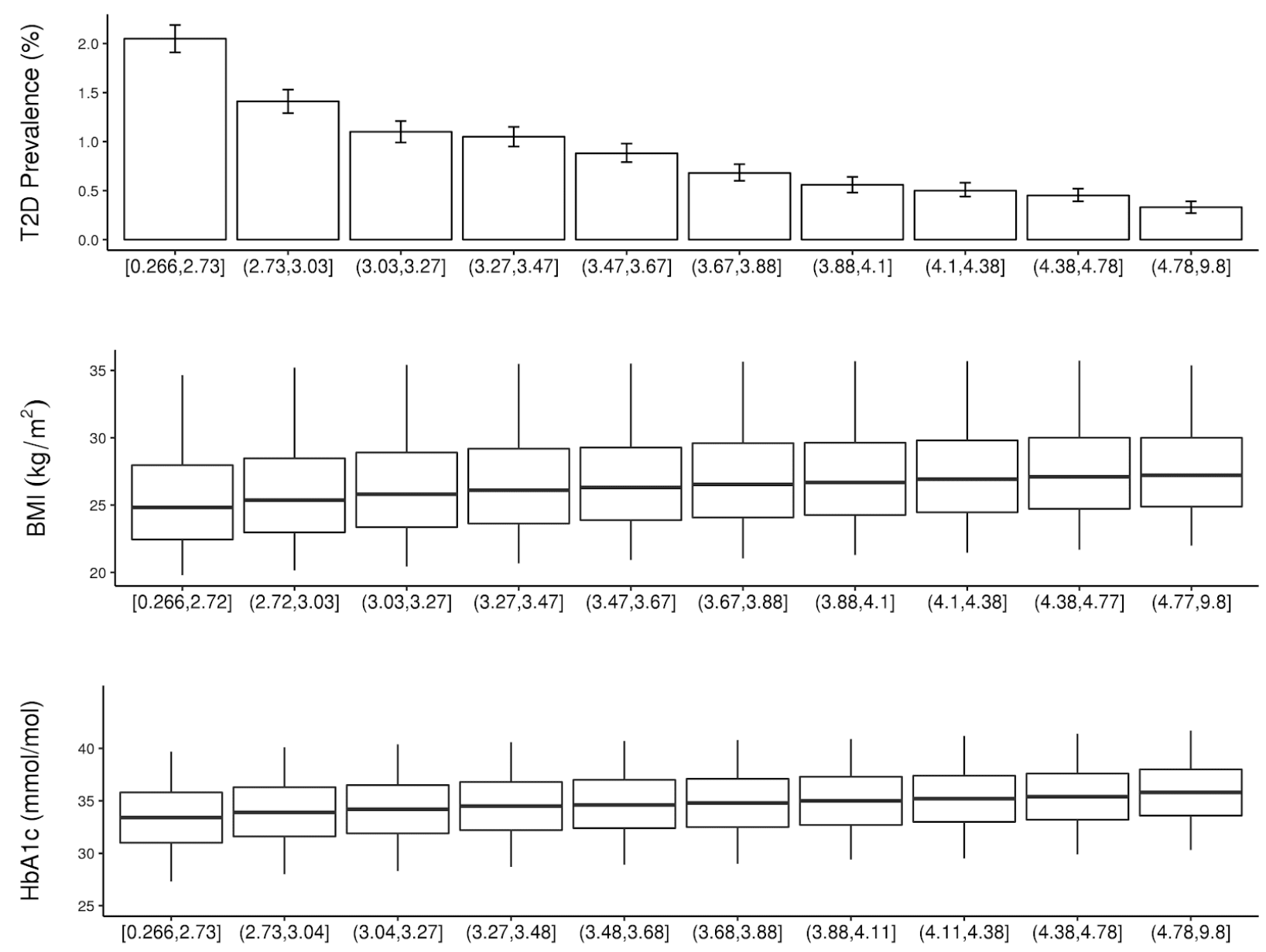
525 Figure 2: Plot of beta coefficients for LDL-C vs. T2D for SNPs with opposite directions of deviations for LDL-C.

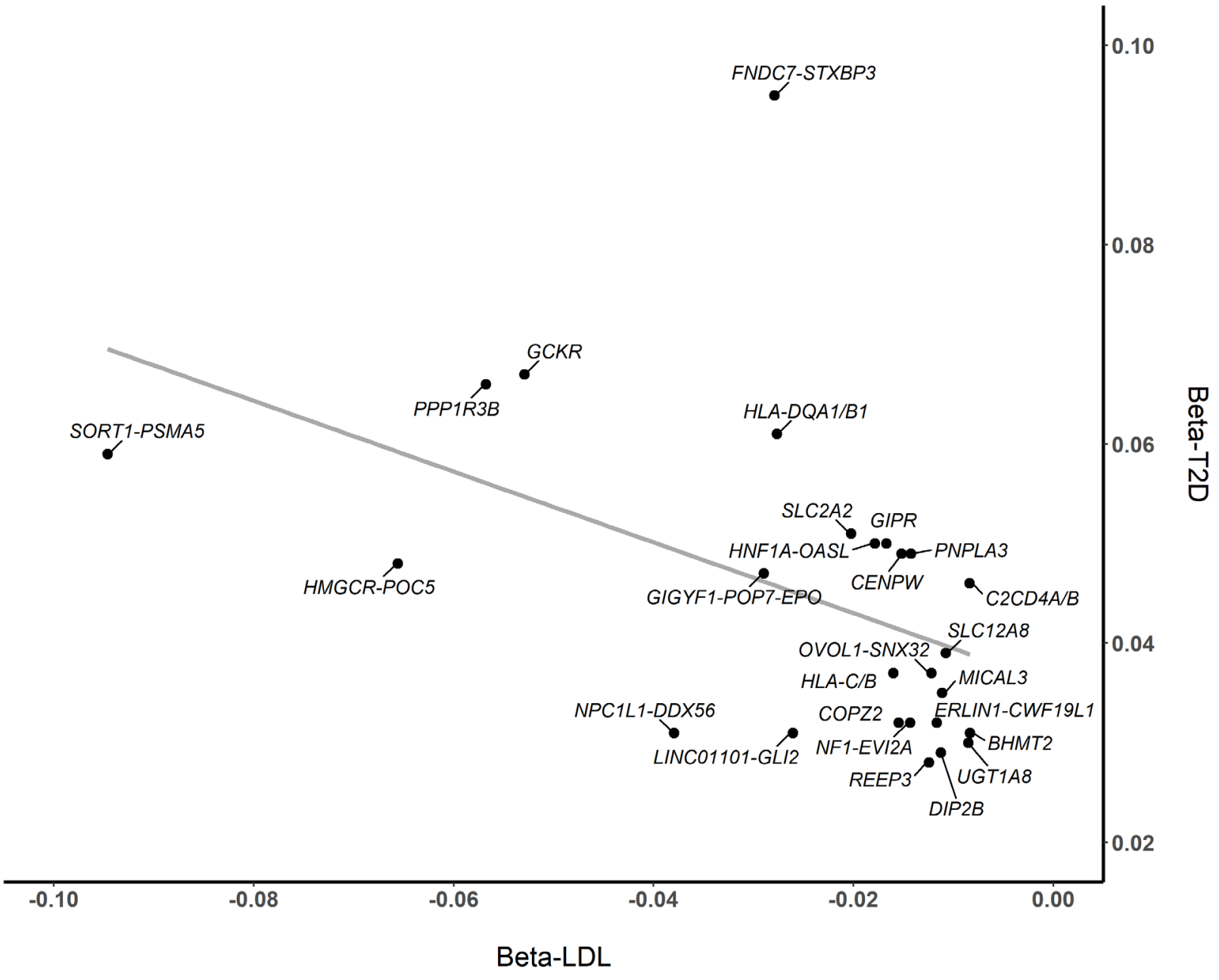


Figure 3: Association of T2D increasing allele at 31 identified SNPs with nine cardiometabolic

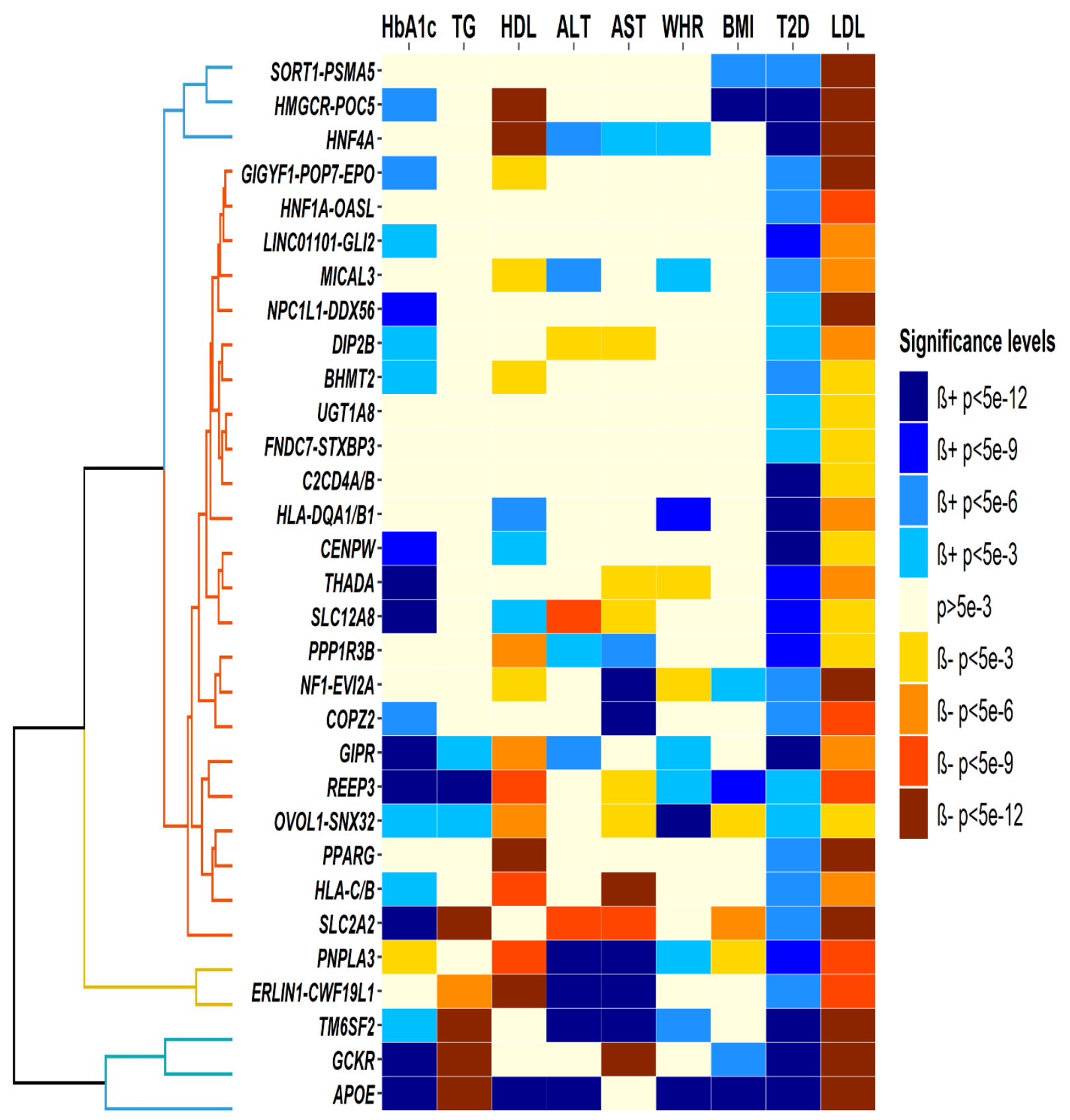

